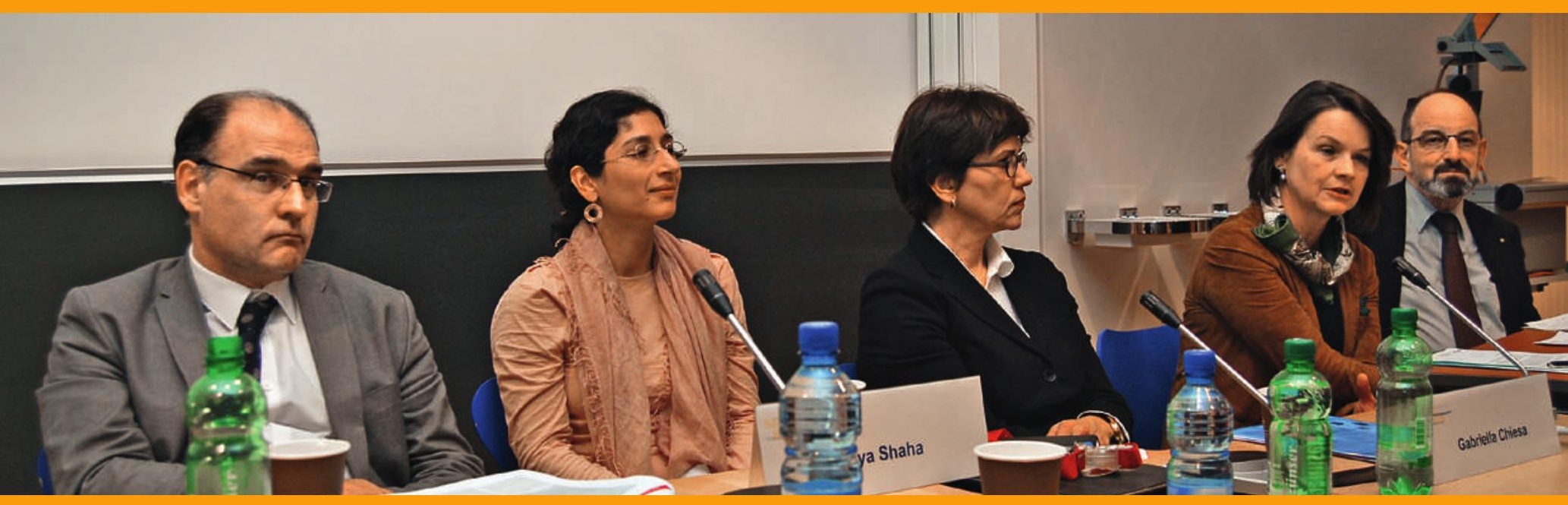

Das Zürcher Podium (v. I.n. r.): Pierre-Alain Clavien, Maya Shaha, Gabriella Chiesa, Christina Brunnschweiler, Ernst Gähler. Fotos: R.B.

SÄZ-Podium zum Thema «Von der Hierarchie zum Team:

Interprofessionalität im Schweizer Gesundheitswesen»

\title{
Mehr kooperieren statt delegieren
}

\author{
Das SÄZ-Podium vom 19. April 2012 in Zürich fokussierte auf das wichtige, in seiner \\ Bedeutung in weiten Kreisen noch unterschätzte Thema «Interprofessionalität im \\ Schweizer Gesundheitswesen». Die angeregte Diskussion bestätigte, dass in diesem \\ Bereich Optimierungsbedarf besteht.
}

Felicitas Witte

Dr. med., freie Medizinjournalistin

Der «Halbgott in Weiss», der der Schwester Anweisungen erteilt - ein gängiges Bild in alten Arztserien. Seitdem hat sich die Zusammenarbeit von Medizinern und Pflegenden geändert. Pflegekräfte haben Verantwortung, durch Spezialisierungen wissen sie mehr als früher und mitunter mehr als die Ärzte etwa im Bereich Dekubitus-Prophylaxe. Doch wie

\section{«Ärzte und Pflegepersonal müssen diskutieren, welche} Aufgaben Ärzte abgeben können, was Pflegende besser können, wo es Überschneidungen gibt und wie eine gute Zusammenarbeit im Team klappen kann.» (E. Wolff)

\footnotetext{
* www.mhiz.uzh.ch/ departments/Museum/ WhoCares.html
}

Korrespondenz: Schweizerische Ärztezeitung Redaktion

Farnsburgerstrasse 8 CH-4132 Muttenz

redaktion.saez[at]emh.ch sieht der Alltag auf Station aus? Arbeiten Pflegende und Ärzte wirklich «auf Augenhöhe», respektieren sie die gegenseitigen Kompetenzen und arbeiten sie im Team zusammen? Solche Fragen diskutierten sechs Experten an der Podiumsdiskussion der Schweizerischen Ärztezeitung am 19. April in Zürich.

\section{Passender Veranstaltungsort}

Der Ort der Veranstaltung war gut gewählt: Man traf sich im Medizinhistorischen Institut der Uni Zürich, wo zurzeit die Wanderausstellung «WHO CARES?» über Geschichte und Gegenwart des Pflegeberufes in Deutschland informiert*. Eine «lebhafte Auseinandersetzung», wie sie an einer Universität üblich sei, wünschte sich Flurin Condrau, Direktor des Medizinhistorischen Instituts und Museums. «Der Pflegeberuf hat sich gewandelt», sagte er. «Pflegende wissen und können viel mehr als früher. Doch immer noch gibt es Ärzte, die ungern Kompetenzen abgeben.» Interprofessionalität im Schweizer Gesundheitswesen sei ein heissumstrittenes Thema, führte Urs Stoffel, Präsident der Ärztegesellschaft des Kantons Zürich, in die Diskussion ein. «Ich hoffe, dass Schnittstellen zwischen den verschiedenen Berufen zu Nahtstellen werden», sagte Stoffel. «Ein Abgeben von Kompetenzen kann auch einen Gewinn an Qualität bedeuten.»

Die klassische Aufgabenverteilung von früher gäbe es nicht mehr, sagte Eberhard Wolff, SÄZRedaktor und Beauftragter Sonderausstellungen des Medizinhistorischen Museums. «Ärzte und Pflegepersonal müssen diskutieren, welche Aufgaben Ärzte abgeben können, was Pflegende besser können, wo es Überschneidungen gibt und wie eine gute Zusammenarbeit im Team klappen kann.» Sowohl in der Pflege als auch in der Medizin gibt es immer mehr Sonderausbildungen. «Wir haben jetzt Dutzende von Experten mit unterschiedlichen Spezialisierun- 


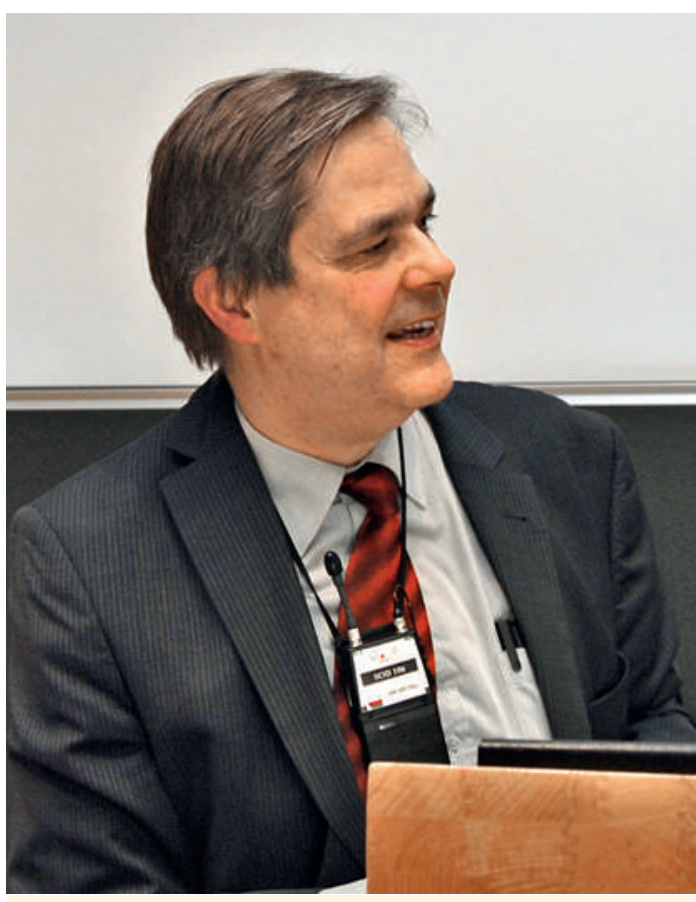

Moderator Eberhard Wolff versuchte, die Diskutierenden gelegentlich mit provokanten Fragen aus der Reserve zu locken. miteinander kommuniziert. «Pflegenden fehlt oftmals noch Selbstbewusstsein. Sie müssen aber wissen, was sie können - manche früher typische ärztliche Handlungen sogar besser als der Arzt.» So gibt es in der Schweiz Pflegefachpersonen mit Master, die Patienten mit kardiovaskulären Problemen bei Lifestyle-Fragen, Umgang mit Medikation, Ernährung, Bewegung und Stress aufklären, die HIV- oder Brustkrebs-Patienten spezifisch beraten oder die sich speziell mit Stomata oder Sturzprävention auskennen.

Reibungspunkte mit Ärzten beobachtet Shaha vor allem dann, wenn man sich über die gemeinsame Vorgehensweise nicht einig sei oder erst gar nicht darüber spräche. «Pflegende planen zum Beispiel schon beim Eintritt des Patienten dessen Ent-

\section{«Jemand muss eine Entscheidung treffen. Wenn viele unterschied- licher Meinung sind und keiner entscheidet, leidet am Ende der Patient.» (Pierre-Alain Clavien)}

lassung und wie er weiter zu Hause versorgt wird», sagte Shaha. «Viele Ärzte haben aber gar keine Vorstellung davon und sind überrascht oder angenervt, wenn wir sie darauf ansprechen.»

\section{Gute Zusammenarbeit spart auch Geld}

Eine gute Zusammenarbeit komme nicht nur dem Patienten zugute, man könne damit auch Geld sparen, sagte Gabriela Chiesa, Ressortleiterin Innovation im Versorgungsmanagement bei der CSS-Versicherung. Sie berichtete von einem Pilotprojekt einer integra-

\section{«Pflegenden fehlt oftmals noch Selbstbewusstsein. Sie müssen aber wissen, was sie können - manche früher typische ärztliche Handlungen sogar besser als der Arzt.» (Maya Shaha)}

\section{Kommunikation ist zentral}

Zentral für eine reibungslose Zusammenarbeit sei die Kommunikation, sagte Maya Shaha, wissenschaftliche Mitarbeiterin in der Direktion Pflege/MTT am Inselspital und Oberassistentin am Institut für Pflegewissenschaft in Lausanne. «Es gibt nichts Schlimmeres, als wenn die Beteiligten sich nicht absprechen und keine gemeinsame Linie fahren.» Hierfür sei es wichtig, dass sowohl Ärzte als auch Pflegende geschult werden, wie man mit den richtigen Worten tiven Versorgung von Asthmapatienten, das kürzlich gestartet wurde. «Bis wir das Konzept hatten, dauerte es zwei Jahre. Alle Beteiligten mussten sich erst kennenlernen und Vorurteile abbauen. Aber jetzt erhält der Asthma-Patient eine koordinierte Betreuung.»

Für einen grossen Hinderungsgrund hält Christina Brunnschweiler, CEO bei der Spitex in Zürich Limmat, das extreme Standesdenken mancher Ärzte. «Während 25 Jahren in der Arbeit in der freien Wirtschaft kannte ich so etwas überhaupt nicht. Ärzte 
«Für mich ist Interprofessionalität keine Neuerfindung, sondern

Alltag.» (Ernst Gähler)

müssen aber lernen zu kooperieren, statt nur zu delegieren.» Immer noch gäbe es Ärzte, die meinen, manches besser zu wissen, etwa bei der Wundpflege. «Dafür haben wir ausgebildete Pflegefachkräfte - die Ärzte müssen uns vertrauen können.»

Ernst Gähler, seit 28 Jahren niedergelassener Allgemeinpraktiker in Herisau und Vizepräsident der FMH, fragt sich täglich, was er delegieren kann und was nicht. «Für mich ist Interprofessionalität keine
Frauen könnten erwiesenermassen besser kommunizieren und seien mehr darauf aus, zu einer Lösung zu kommen.

Neben dem Geschlecht kann der «menschliche Faktor» die Zusammenarbeit behindern. «Ob mein Wissen akzeptiert wurde oder nicht, hing immer vom Gegenüber $a b »$, berichtete eine Frau aus dem Publikum, die 30 Jahre als Pflegefachfrau arbeitete. «Im Alltag war das sehr ermüdend.» Ob Regeln oder Standards dies ändern könnten, ist fraglich. «Reglementierung behindert», sagte Christina Brunnschweiler von der Spitex. «Wir haben zum Beispiel keine Chance, eine Therapieanpassung zu machen, und müssen uns immer beim Arzt rückversichern auch mitten in der Nacht.» Von einer wirklich guten interprofessionellen Zusammenarbeit sei man noch weit entfernt, meinte ein Mann aus dem Publikum,

\section{«Wir müssen die Leute dort einsetzen, wo sie kompetent sind - das ist besonders wichtig bei der Versorgung chronisch Kranker.» (Gabriella Chiesa)}

Neuerfindung, sondern Alltag.» Wichtig sei aber, dass einer den Überblick habe, die Vorgehensweise koordiniere und die Verantwortung trage - und das sei der Hausarzt. «Wir Ärzte müssen aber die Kompetenzen der anderen respektieren und ihnen Aufgaben überlassen, die sie besser können.» Er würde sich zum Beispiel nie anmassen, chronische Wunden zu versorgen. Auch wenn eigentlich eine Untersuchung bei einem schwerkranken Patienten medizinisch notwendig wäre, seine Mitarbeiterin aber meint, der Patient sei zu krank dafür, hört er auf ihren Rat. «Am besten bespricht man die Vorgehensweise dann gemeinsam und bindet den Patienten mit ein.»

In Ländern wie den USA klappt die Zusammenarbeit zwischen Ärzten und Pflegenden anscheinend schon seit Jahren besser. «Ich war geschockt, als ich aus den USA zurückkam», erinnert sich Pflegewissenschafterin Shaha. «Dort wurde meine Kompetenz immer akzeptiert und respektiert und ich wurde nie wie hier mitunter gefragt: Kannst $\mathrm{Du}$ das überhaupt?» Einige Ärzte stellten sich unter dem TeamBegriff immer noch etwas anderes vor als Pflegende. «Wir gehen von einer Ebene auf Augenhöhe aus, manche Ärzte scheinen das anders zu sehen.»

\section{Frauen-Männer-Problematik?}

Im Podium diskutierten zwei Männer in ärztlichen Berufen und drei Frauen mit nichtärztlicher Ausbildung. Ob es eine Frauen-Männer-Problematik sei, dass die Zusammenarbeit zwischen Ärzten und Pflegenden manchmal nicht so gut funktioniere, fragte Redaktor Wolff provokant die Diskutierenden. «Ich hätte gerne mehr Frauen im Team», sagte Clavien. der als Pflegefachkraft in der Psychiatrie arbeitet. «Wir müssen die Zuständigkeiten neu aufteilen und festlegen.» Schon im Studium müssten Ärzte und Pflegende lernen, wer was machen darf.

«Wir müssen die Leute dort einsetzen, wo sie kompetent sind - das ist besonders wichtig bei der

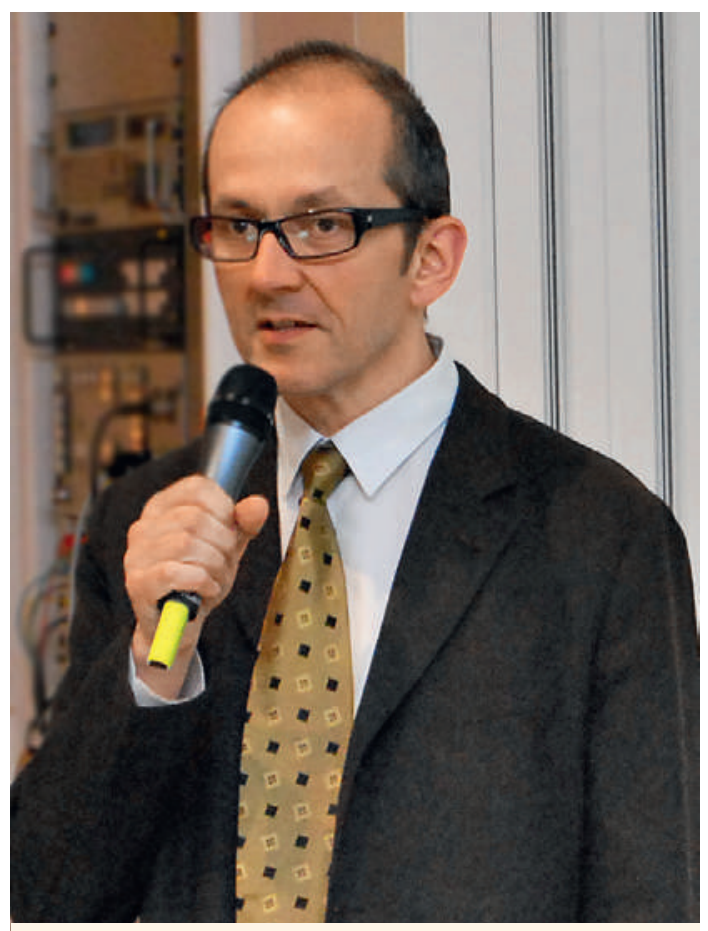

Wünschte sich eine lebhafte Auseinandersetzung: Flurin Condrau, Direktor Medizinhistorisches Institut und Museum und Gastgeber der Veranstaltung. 


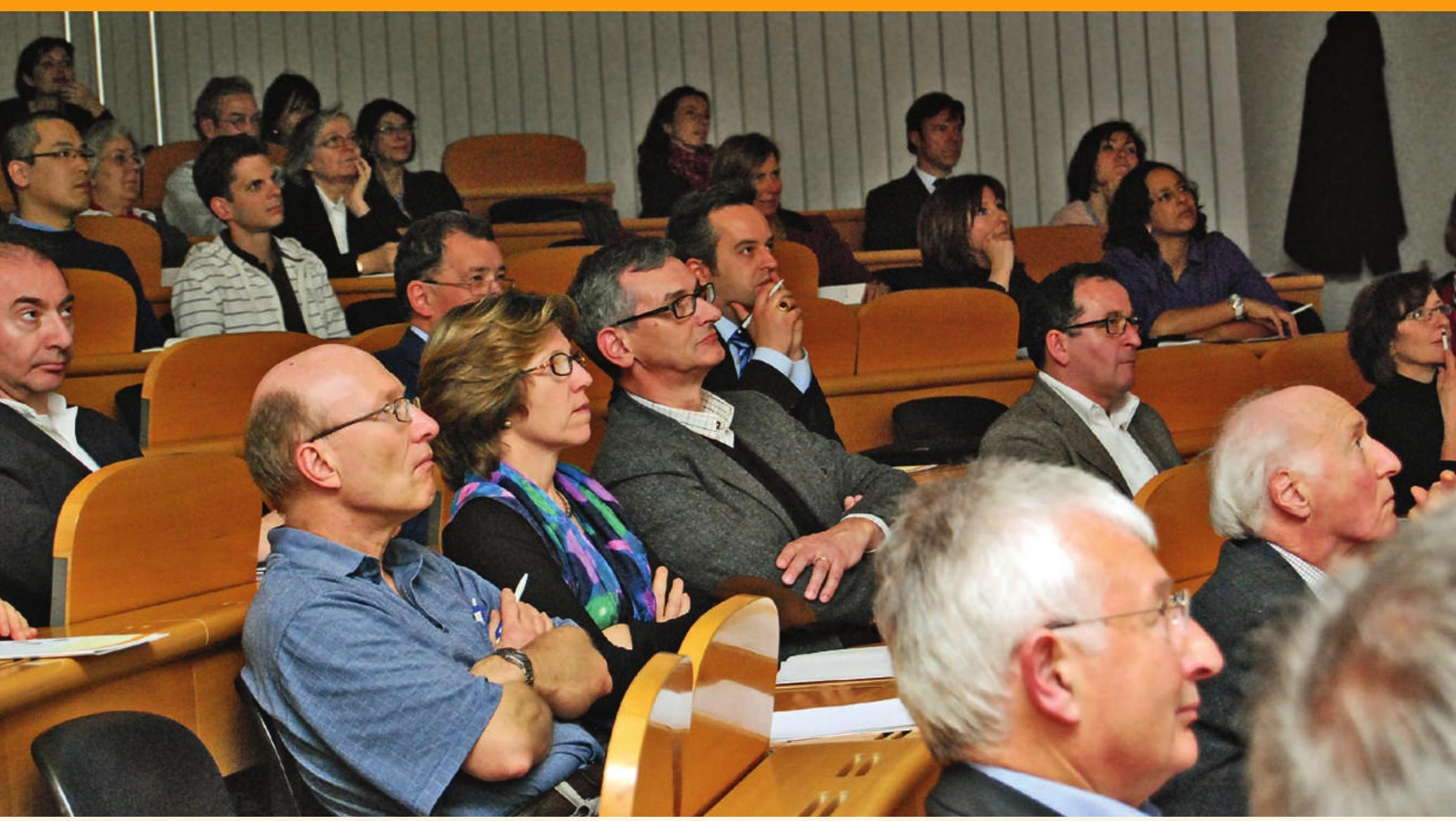

Interessiertes Publikum: Viele Anwesende brachten eigene Erfahrungen in die Diskussion ein.

Versorgung chronisch Kranker», forderte Gabriella Chiesa. Ihre Vision wäre, dass der Patient im Mittelpunkt stünde und die Fäden ziehe. «Er muss es aber können und wollen und ausserdem muss er gut geschult sein.» Hier erlebe sie von manchen Ärzten Ablehnung, die meinen, dass das dem Patienten «doch rasch erzählt sei», und er bräuchte keine Schulung.

Hausarzt Gähler sieht hier neue Berufsmöglichkeiten. «In Zukunft könnten Praxisassistenten oder Pflegefachkräfte zusätzlich in «Chronic Care» ausge-
Beispiel nicht morgens um sieben bei der SpitexPflegekraft an, während sie beim Patientenbesuch ist, und sie nicht montags vormittags bei mir, weil sie weiss, dass dann die Praxis voll ist.»

Auch wenn in vielen Kliniken Ärzte und Pflegende schon besser zusammenarbeiten als zu Zeiten der «Schwarzwaldklinik»: «Noch ist nicht geklärt, welche Bereiche bei der interprofessionellen Zusammenarbeit man schriftlich festlegen kann und sollte und welche Rolle der «menschliche Faktor»spielen

\section{«Wir haben zum Beispiel keine Chance, eine Therapieanpassung zu machen und müssen uns immer beim Arzt rückversichern - auch mitten}

in der Nacht.» (Christina Brunnschweiler)

bildet werden und Schulungen halten. Dann kann sich der Arzt mehr auf seine ärztlichen Tätigkeiten konzentrieren.» Wichtig für eine gute Zusammenarbeit sei, sich in die Mitarbeiter hineinzuempfinden, so ein Hausarzt aus dem Publikum. «Ich rufe zum darf», sagte Flurin Condrau. «Ausserdem sollten wir uns fragen, was ein gutes Team überhaupt bedeutet und wie man zu einem guten Team wird.» Hierzu wird es sicherlich noch weiterer lebhafter Diskussionen bedürfen. 